\title{
Different length scales for order parameters in two-gap superconductors: Extended Ginzburg-Landau theory
}

\author{
L. Komendová, M. V. Milošević, A. A. Shanenko, and F. M. Peeters \\ Departement Fysica, Universiteit Antwerpen, Groenenborgerlaan 171, B-2020 Antwerpen, Belgium
}

(Received 31 March 2011; revised manuscript received 21 July 2011; published 24 August 2011)

\begin{abstract}
Using the Ginzburg-Landau theory extended to the next-to-leading order, we determine numerically the healing lengths of the two order parameters at the two-gap superconductor/normal metal interface. We demonstrate on several examples that those can be different even in the strict domain of applicability of the Ginzburg-Landau theory. This justifies the use of this theory to describe relevant physics of two-gap superconductors, distinguishing them from their single-gap counterparts. The calculational degree of complexity increases only slightly with respect to the conventional Ginzburg-Landau expansion, thus the extended Ginzburg-Landau model remains numerically far less demanding compared to the full microscopic approaches.
\end{abstract}

DOI: 10.1103/PhysRevB.84.064522

PACS number(s): 74.20.De, 05.30.Fk, 74.70.Ad, 74.70.Xa

\section{INTRODUCTION}

Over the past half century, the Ginzburg-Landau (GL) theory ${ }^{1}$ has proven to be a very helpful tool in studies of superconductors, but also other systems in and out of the condensed-matter physics. By its construction, the formalism is only justified near the critical temperature $T_{c}$, but it typically produces qualitatively correct results even far below the $T_{c}$. Recently an exception was found to this unwritten rule, when it was shown that the standard formulation of the GL theory is insufficient for adequate description of two-band (or multiband) superconductors because it predicts the same spatial variation of the condensates in all bands ${ }^{2}$ (within its range of applicability, i.e., close to $T_{c}$ ). This renders it unable to connect to the theoretical results obtained by using Usadel ${ }^{3,4}$ or Eilenberger ${ }^{5}$ equations in the broader temperature range, which unambiguously show the presence of two separate length scales for two gaps. Experimentally, the evidence for different coherence lengths in $\sigma$ and $\pi$ bands of $\mathrm{MgB}_{2}$ was previously found by direct vortex imaging, ${ }^{6}$ muon spin relaxation ( $\mu \mathrm{SR})$ imaging of the supercarrier density, ${ }^{7}$ and in pronounced features in the flux-flow resistivity ${ }^{8}$ as a function of applied magnetic field.

In order to capture the important physics of different length scales, one needs to extend the GL formalism as realized in Ref. 9, where the two order parameters are calculated up to order $\tau^{3 / 2}$ in the small deviation from the critical temperature $\tau=1-T / T_{c}$, instead of the standard $\tau^{1 / 2}$ as used in Ref. 2. In the latter paper, the authors mention that the extra terms in the next-to-leading order are by construction small corrections, not significant enough to alter the single coherence length controlling the spatial distribution of both condensates. First, we argue that the effects of higher-order corrections can be significant since the above argument about small corrections applies only to the order parameter-not necessarily to its spatial profile, i.e., healing lengths and other physical quantities. Second, we argue that any (even small) difference between the characteristic length scales of two condensates is of fundamental importance, since it may lead to other unique phenomena related to the competition of length scales. Note that here we do not enter the recent debate about sufficient discrepancy of length scales to provide "type-1.5" superconductivity. ${ }^{10-12}$ Instead, we complement that discussion by exactly quantifying the difference in length scales in the domain of the extended GL theory.

The fact that the difference of characteristic length scales of two Cooper pair condensates exists even in the strict Ginzburg-Landau domain is of great practical importance, since the calculations based on the Ginzburg-Landau theory are typically far less computationally demanding than the calculations based on full microscopic theories (Bogoliubovde Gennes, Gor'kov, Usadel, or Eilenberger equations). In microscopic formalisms, one usually has to make clever approximations and make compromises in the calculation procedure. As a consequence, even though these approaches are valid in the whole temperature range from absolute zero to $T_{c}$, they are limited to the simple systems such as a single vortex or other highly symmetric or effectively one-dimensional cases. On the contrary, the Ginzburg-Landau equations have a much simpler structure and therefore allow for studying of highly nontrivial situations such as vortex lattice statics and dynamics, current-driven systems, and interaction with pinning and fluxonic devices. Since the above is well established in the standard GL formalism, we emphasize here that the calculations become only a fraction more complicated in the extended Ginzburg-Landau formalism-while it does contain more equations (for the order parameters to the leading and next-to-leading order), the coupling of the equations for different order parameters is realized through the coefficients and not the calculated variables - contrary to the standard set of two-band GL equations. ${ }^{12,13}$

Finally, the present paper and its conclusions are relevant not only to bulk multiband materials, but also to nanoscale superconductors, e.g., single-crystalline metallic nanofilms, ${ }^{14-17}$ and also pancake-shaped superfluid Fermi gases in optical traps, ${ }^{18}$ where the multiband structure appears due to quantum confinement. The key mechanism there is the formation of discrete single-particle energy levels for the motion in the strongly confined direction (while motion is quasifree in the other directions).

\section{THEORETICAL FORMALISM}

Following Ref. 9, we employ the extended GL formalism for the order parameters $\Delta_{j}$ evaluated up to order $\tau^{3 / 2}$ by 
taking $\Delta_{j}(\mathbf{x})=\Delta_{j}^{(0)}(\mathbf{x})+\Delta_{j}^{(1)}(\mathbf{x})$, where $\Delta_{j}^{(0)}(\mathbf{x}) \propto \tau^{1 / 2}$ and $\Delta_{j}^{(1)}(\mathbf{x}) \propto \tau^{3 / 2}$, with $j=1,2$ indexing two coupled condensates in a two-band superconductor. In the absence of applied magnetic field, the order parameters can be taken real. The extended GL equations then read (at zero magnetic field)

$$
\begin{gathered}
\alpha \Delta_{j}^{(0)}+\beta_{j}\left[\Delta_{j}^{(0)}\right]^{3}-K \nabla^{2} \Delta_{j}^{(0)}=0, \\
\Delta_{j}^{(1)}\left(\alpha+3 \beta_{j}\left[\Delta_{j}^{(0)}\right]^{2}\right)-K \nabla^{2} \Delta_{j}^{(1)}=F\left(\Delta_{j}^{(0)}\right)+F_{j}\left(\Delta_{j}^{(0)}\right),
\end{gathered}
$$

where $\quad \alpha=-N(0) \tau\left(n_{1} \mathcal{A}_{2}+n_{2} \mathcal{A}_{1}\right) / \lambda_{12}, K=N(0) \hbar^{2} W_{3}^{2}$ $\left(n_{1} v_{1}^{2} \mathcal{A}_{2}+n_{2} v_{2}^{2} \mathcal{A}_{1}\right) /\left(6 \lambda_{12}\right), \beta_{1(2)}=N(0) W_{3}^{2}\left(n_{1(2)} \mathcal{A}_{2(1)} / \lambda_{12}+\right.$ $\left.n_{2(1)} \mathcal{A}_{1(2)}^{3} / \lambda_{12}^{3}\right)$, with $\mathcal{A}_{1}=\lambda_{22}-\eta n_{1} \mathcal{A}$ and $\mathcal{A}_{2}=\lambda_{11}-$ $\eta n_{2} \mathcal{A}$. Here $\mathcal{A}=\ln \left(2 e^{\Gamma} \hbar \omega_{D} / \pi T_{c}\right)$, with Euler constant $\Gamma=0.577$ and $\omega_{D}$ the Debye frequency, and $\eta$ denotes the determinant of the interaction matrix $\lambda_{i j}=N(0) g_{i j}$, with $g_{i j}$ the coupling constant, $N(0)$ the total density of states (DOS), and $n_{j} N(0)$ the band-dependent DOS. In addition, the coefficients feature Fermi velocities $v_{j}$ of both bands, $W_{3}^{2}=\frac{7 \zeta(3)}{8 \pi^{2} T_{c}^{2}}$, with $\zeta(\cdots)$ the Riemann zeta function, and terms $F$ and $F_{j}$ which are given in complete form in Ref. 9. Since Eq. (1a) is completely equivalent to the single-gap GL equation and the bands share the same critical temperature $T_{c}$, we have either both bands normal or both bands superconducting. In the first case, Eq. (1b) allows only $\Delta_{j}^{(1)}=0$ as a solution and thus superconductivity cannot be restored by corrections in the extended GL model. In the following we will consider that both bands are superconducting and their bulk amplitudes $W_{j}=\sqrt{-\alpha / \beta_{j}}$ are real. We rescale the equations using $\Delta_{j}^{(k)}=W_{j} \widetilde{\Delta}_{j}^{(k)}$ and $x=\xi \tilde{x}$, where $\xi=\sqrt{-K / \alpha}$ is the length unit common for both condensates, and obtain (tildes omitted)

$$
\begin{gathered}
\Delta_{j}^{(0)}-\left[\Delta_{j}^{(0)}\right]^{3}+\nabla^{2} \Delta_{j}^{(0)}=0 \\
\Delta_{j}^{(1)}\left(1-3\left[\Delta_{j}^{(0)}\right]^{2}\right)+\nabla^{2} \Delta_{j}^{(1)}=\frac{F\left(\Delta_{j}^{(0)}\right)+F_{j}\left(\Delta_{j}^{(0)}\right)}{\alpha W_{j}} .
\end{gathered}
$$

The first equation is the same for both bands, while the second one differs through the terms on the right-hand side. This is exactly the cause for the emergence of two different characteristic lengths in the two bands. From Eq. (2) it is also directly apparent why this does not happen in the order parameters of order $\tau^{1 / 2}$, i.e., in Eq. (2a), but only in the higher-order considerations as in Eq. (2b). To evaluate the coefficients entering Eq. (2), one needs in principle to specify the coupling constants $\lambda_{11}, \lambda_{22}$, and $\lambda_{12}$, the partial density of states in one band, e.g., $n_{1}$ (since $n_{1}+n_{2}=1$ this determines $n_{2}$ as well), and the ratio of the Fermi velocities $v_{1} / v_{2}$. All other parameters enter units of scaling, and therefore have no impact on any physical effects.

\section{THE GINZBURG-LANDAU DOMAIN}

Before discussing the numerical results of Eq. (2), it is very important to get a feeling about the relevant values of $\tau$, i.e., the domain of applicability of the extended GL model. First of all, our analysis shows that $\tau \Delta_{j}^{(0)} \sim \Delta_{j}^{(1)}$. Then, by construction, $\Delta_{j}^{(0)}>\Delta_{j}^{(1)}$ and the trivial inequality $\tau<1$ holds. However, this is a necessary but not sufficient condition justifying Eq. (2), as one also needs to keep in mind the justification for the use of the gradient expansion in the derivation of Eq. (2). This expansion requires the GL coherence length $\xi$ to be larger than the band-dependent correlation length $\zeta_{j}=\hbar v_{j} /(2 \pi T)$ that controls the spatial variations of the relevant kernels in the integral expansion of the anomalous (Gor'kov) Green's function in powers of the band-dependent order parameters (in the clean limit), i.e.,

$$
\zeta_{1}, \zeta_{2}<\xi
$$

It is important to note here that for a two-band superconductor $\zeta_{j}$ is not necessarily close to the band-dependent generalization of the Pippard length, i.e., $\hbar v_{j} /\left.\left(\pi \Delta_{j}\right)\right|_{T=0}$, often used as an estimate of the coherence lengths of two bands. ${ }^{2,11}$ Using definitions of $\alpha, K$, and $\beta_{j}$, we obtain from Eq. (3) the following estimate for the GL domain $\tau \lesssim \tau^{*}$ with

$$
\frac{\tau^{*}}{\left(1-\tau^{*}\right)^{2}}=1+\frac{v_{1}^{2} / v_{2}^{2}-1}{1+n_{2} \mathcal{A}_{1} /\left(n_{1} \mathcal{A}_{2}\right)} \quad\left(v_{1}<v_{2}\right) \text {. }
$$

If $v_{1} \geqslant v_{2}$, the replacement $1 \leftrightarrow 2$ should be made. Equation (4) shows that the extent of the Ginzburg-Landau domain for specific multigap superconductor depends on its material parameters, as was first pointed out in Ref. 4. Equation (4) is most sensitive to the ratio of the Fermi velocities $v_{1} / v_{2}$. In particular, when $v_{1} / v_{2}=1$, the above inequality simply implies $\tau \lesssim 0.38$, i.e., the same as in the one-band case, regardless of the particular values of the other parameters, i.e., $n_{2} / n_{1}$ and $\mathcal{A}_{2} / \mathcal{A}_{1}$ (where $\mathcal{A}_{2} / \mathcal{A}_{1}=\left[\Delta_{1}^{(0)} / \Delta_{2}^{(0)}\right]^{2}$ ). For $n_{1} \approx n_{2}$ and $\mathcal{A}_{1} \approx \mathcal{A}_{2}$, we typically obtain $0.27<\tau^{*}<0.38$, thus the temperature domain of GL theory is still very large. The GL domain shrinks significantly only when the ratio $n_{2} \mathcal{A}_{1} /\left(n_{1} \mathcal{A}_{2}\right)$ acquires either extremely large or very small values, which, e.g., can occur when the two energy gaps differ more than an order of magnitude and at the same time $v_{1}$ is very different from $v_{2}$. For example, for the magnesium diboride parameters (as elaborated further below) one finds from Eq. (4) $\tau^{*}=0.08-0.32$ depending on the relevant ratio of the Fermi velocities, i.e., on the direction considered, because the $\sigma$ band of magnesium diboride is highly anisotropic. The threshold temperature for the applicability of the GL theory $\left(\tau^{*}\right)$ for a broader range of parameters (i.e., other materials) is plotted in Fig. 1.

\section{DIFFERENT LENGTH SCALES IN SELECTED BORIDES, PNICTIDES, AND NANOTHIN LEAD FILMS}

To evaluate and compare the spatial distribution of the two order parameters in a two-band superconductor, we study the simple case of a superconductor/normal metal $(\mathrm{S} / \mathrm{N})$ interface in the absence of any applied magnetic field. It then suffices to consider the one-dimensional version of Eq. (2), along the $x$ axis perpendicular to the $\mathrm{S} / \mathrm{N}$ interface. The appropriate boundary conditions are $\Delta_{j}^{(k=0,1)}(x=0)=0$, and $\nabla \Delta_{j}^{(k)}(x \rightarrow \infty)=0$, where thus $x=0$ lies at the interface, with superconductivity fully suppressed there. The second boundary condition ensures unperturbed two-gap superconductivity away from the $\mathrm{S} / \mathrm{N}$ interface. In Fig. 2(a) we show the results for $\Delta_{j}(x)$ in $\mathrm{MgB}_{2}$ (normalized to bulk values, at 


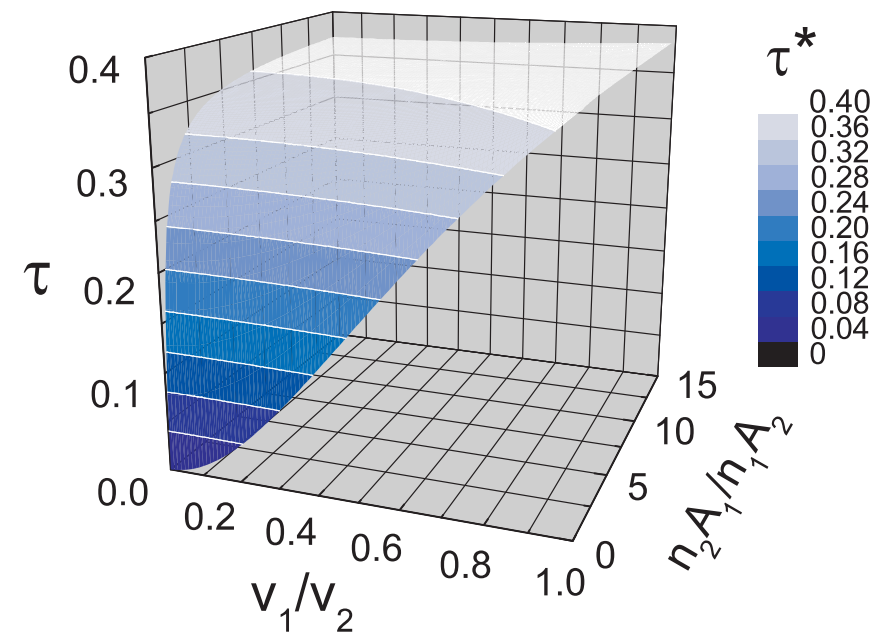

FIG. 1. (Color online) The estimated temperature range $\tau<\tau^{*}$ of the validity of the GL theory based on Eq. (4). The gradient expansion made in the derivation of the GL equations (both standard and extended) is fully justified below the plotted three-dimensional surface.

temperature $T=0.95 T_{c}$, and with other parameters taken from Refs. 11 and 19), obtained within standard, ${ }^{12}$ extended, ${ }^{9}$ and reduced ${ }^{2} \mathrm{GL}$ formalism. In the case of $\mathrm{MgB}_{2}$, the extended GL model clearly gives two length scales for two order parameters $\Delta_{j=1,2}$, smaller than those obtained in the standard GL theory (with incomplete higher-order terms).

For correct comparison one must define a measure for the spatial variations of $\Delta_{j}$. From the numerical solution of

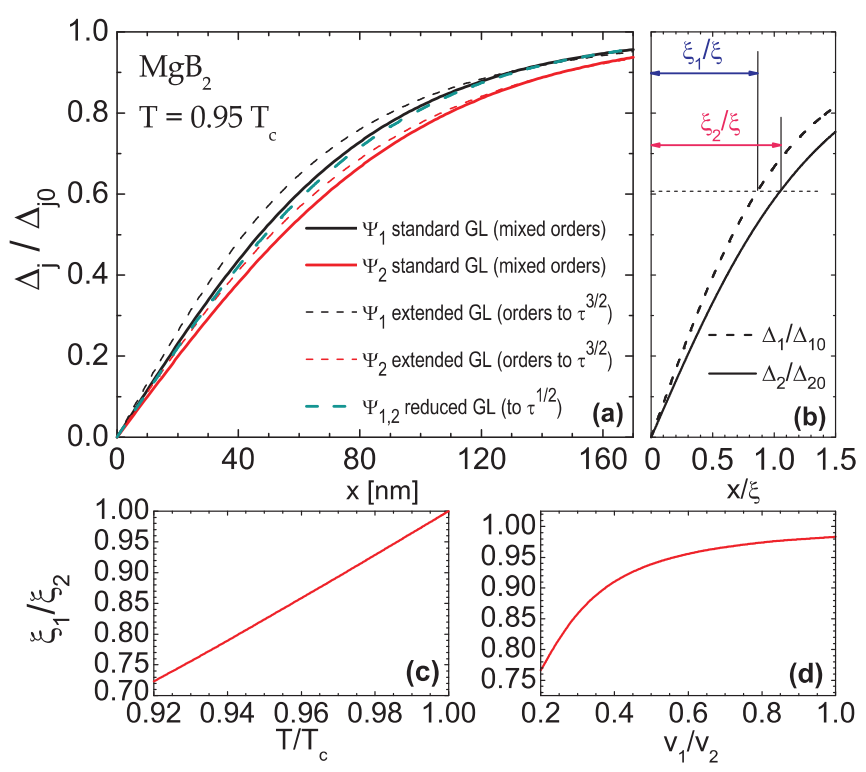

FIG. 2. (Color online) (a) The spatial profile of the order parameters at the $\mathrm{S} / \mathrm{N}$ interface at $T=0.95 T_{c}$, for microscopic parameters of $\mathrm{MgB}_{2}\left[\xi_{1} / \xi_{2}=v_{1} / v_{2}=0.255\right.$ (Ref. 11$) ; \lambda_{11}=1.88, \lambda_{22}=$ $0.5, \lambda_{12}=0.21, n_{1}=0.43$ (Ref. 19)], compared in three versions of the two-band GL theory. The ratio of the healing lengths of the two coupled condensates $\xi_{1} / \xi_{2}$ calculated in extended GL theory [with $\xi_{1,2}$ determined as illustrated in (b)] is shown in (c) as a function of temperature for fixed $v_{1} / v_{2}=0.255$, and in (d) as a function of the ratio $v_{1} / v_{2}$ at $T=0.95 T_{c}$.
Eq. (2a) we found that at the characteristic distance $\xi$ the single-gap-like order parameter $\Delta_{j}^{(0)}$ increases from zero to 0.6089 of its bulk value. Therefore, we define the healing lengths $\xi_{j}$ for the two condensates in a two-gap superconductor using the criterion $\Delta_{j}\left(\xi_{j}\right) \equiv 0.6089 \Delta_{j 0}$, where $\Delta_{j 0}$ is the bulk value of $\Delta_{j}$, as depicted in Fig. 2(b). The difference between the characteristic length scales in two bands is clear already from their definition in Fig. 2(b), but we emphasize this point in Figs. 2(c) and 2(d), where the ratio of the healing lengths of the two order parameters is plotted as a function of temperature [Fig. 2(c)] and ratio of Fermi velocities $v_{1} / v_{2}$ [Fig. 2(d)]. At the lowest temperature shown in Fig. 2(c), $T=0.92 T_{c}$, the disparity between the healing lengths is already over $25 \%$, and the difference increases as the ratio of the Fermi velocities is taken smaller [see Fig. 2(d)].

In the analysis of the above phenomenon we noticed that only the coefficient $S$ in front of the term $\nabla^{2} \Delta_{j}^{(0)}$ in $F\left(\Delta_{j}^{(0)}\right)$ depends both on $\tau$ and $v_{1} / v_{2}$ :

$$
S=\frac{\hbar^{2} N(0) W_{3}^{2} \tau}{6 \lambda_{12}} \sum_{j=1,2} v_{j}^{2}\left[2 n_{j} \lambda_{j j}-\eta n_{1} n_{2}(1+2 \mathcal{A})\right] .
$$

Due to this special form, for particular values of $n_{j}$ and $\lambda_{i j}$ the term in brackets can be positive for one band, but negative for the other. In such a case it is possible to change the sign of $S$ term by varying the ratio of the Fermi velocities $v_{1} / v_{2}$ around its threshold value

$$
\left(\frac{v_{1}}{v_{2}}\right)^{*}=\left(-\frac{2 n_{2} \lambda_{22}-\eta n_{1} n_{2}(1+2 \mathcal{A})}{2 n_{1} \lambda_{11}-\eta n_{1} n_{2}(1+2 \mathcal{A})}\right)^{1 / 2},
$$

which equals 0.46 for the parameters of Fig. 2. Therefore, two cases with $v_{1} / v_{2}$ significantly larger and smaller than 0.46 will show very different behavior with respect to the disparity of the healing lengths of the two condensates, as is visible in Fig. 2(d). For $v_{1} / v_{2} \approx 0.46$, the term with coefficient $S$ has no influence and other terms determine the spatial behavior of the order parameters.

Besides the difference in the healing lengths of the two condensates, the extended GL model provides insight also in a more accurate temperature dependence of the order parameters. Namely, standard GL theory leaves one with simply $\Delta \propto \tau^{1 / 2}$, which is shown in Fig. 3 to be clearly inadequate for our calculations. Specifically, in Fig. 3 we show two healing lengths as a function of temperature, both already normalized to the temperature-dependent length $\xi \propto \tau^{-1 / 2}$. It is directly obvious that we are left with a nonconstant value, and we fitted the general temperature dependence of the residue to a quadratic form $\xi_{j} / \xi=1+A \tau+B \tau^{2}$, as shown in Fig. 3. Note that the accurate fitting of our data requires a quadratic function, although the terms of order $O\left(\tau^{2}\right)$ are not included in the extended GL theory. Therefore, the obtained coefficient $B$ should be taken with reservations.

In what follows, we extend our analysis to materials other than $\mathrm{MgB}_{2}$. We first address the recently discovered ironpnictides, more specifically LiFeAs. Interestingly enough, several existing experimental works on this material provide us with radically different microscopic parameters obtained from the fit of the superfluid density data in the self-consistent $\gamma$ model $^{20,21}$ and from two-band fitting of the upper critical field. ${ }^{22,23}$ In Fig. 4(a), we show the GL-calculated ratio of the 


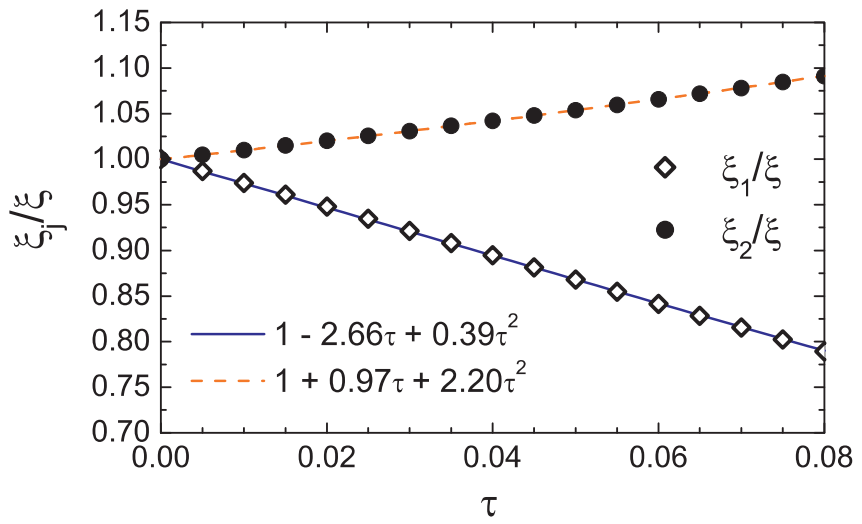

FIG. 3. (Color online) The healing lengths $\xi_{1}$ (empty rhombuses) and $\xi_{2}$ (solid circles) of the two order parameters in $\mathrm{MgB}_{2}$ [Fig. 2(a)] scaled to the standard GL length scale $\xi$ as a function of temperature. The solid (dashed) line shows the fitting function of $\xi_{1} / \xi\left(\xi_{2} / \xi\right)$.

healing lengths of two bands in LiFeAs, for the parameters taken from latter two references. Although different, both curves in Fig. 4 clearly show a discrepancy between the healing lengths, up to $20 \%$ at temperatures above $0.8 T_{c}$.

Specific bulk superconductors are not the only possible multiband system. As already mentioned in the Introduction, other interesting examples are single-crystalline superconducting nanofilms and quasi-1D and 2D optically trapped fermionic condensates. An advantage of such multiband systems is that the band Fermi velocities can be widely tailored here by simply changing the quantum-confinement dimensions (through a change in the energetic positions of the discrete levels-see details in Ref. 24). In particular, the $\mathrm{Pb}(111)$ nanofilms with thickness four and five monolayers (ML) are two-band superconductors due to the presence of only two perpendicular single-electron levels below the Fermi energy. ${ }^{16}$ In this case, the ratio of the band Fermi velocities can be estimated on the basis of the available tunneling data in Ref. 16, i.e., the energetic positions of single-electron levels. This results in $v_{1} / v_{2} \approx 3$ or $v_{1} / v_{2} \approx 2.5$ for 4 and $5 \mathrm{ML}$, respectively (when assuming the parabolic band approximation for each band with the same band mass). In addition, we have the constraint

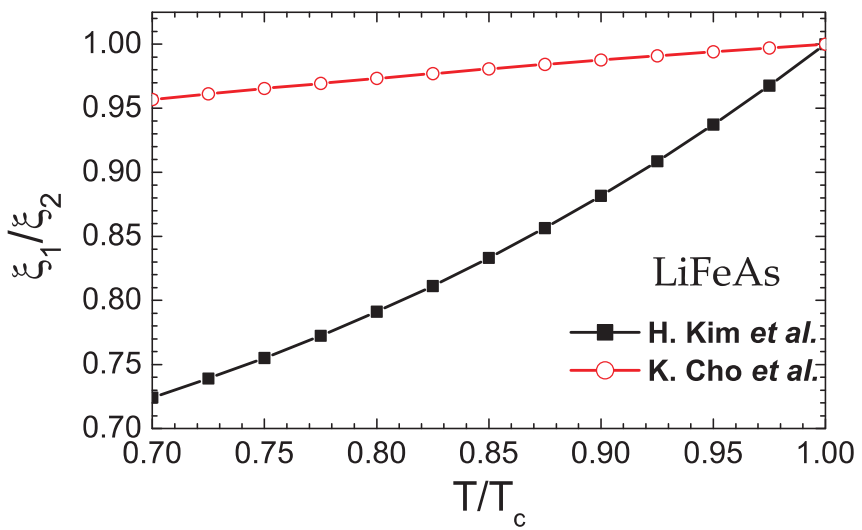

FIG. 4. (Color online) The temperature dependence of the ratio of the healing lengths $\xi_{1} / \xi_{2}$ in LiFeAs, for parameters from Ref. 21 (solid squares) and Ref. 22 (empty circles).

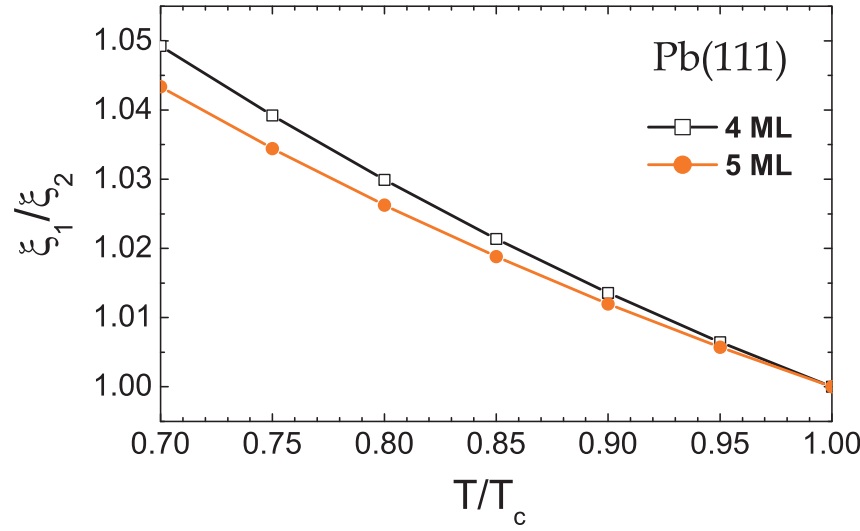

FIG. 5. (Color online) The temperature dependence of the ratio of the healing lengths $\xi_{1} / \xi_{2}$ in 4- (open squares) and 5-ML-thick (solid circles) $\mathrm{Pb}(111)$ nanofilms.

$\lambda_{11}=\lambda_{22}=1.5 \lambda_{12}$, the ratio between the interband and intraband coupling which is typical for the film geometry. ${ }^{25,26}$ The thickness-dependent $T_{c}$ can then be taken to adjust $\lambda_{11}$, which is expected to be close to its bulk value 0.39 . For the band-dependent dimensionless DOS it is reasonable to take $n_{1}=n_{2}$, which follows from the well-known expression for the two-dimensional DOS. We inserted such estimated parameters in the extended GL formalism, and again obtained a nonzero difference between the spatial scales of the two order parameters $(\sim 5 \%)$, as shown in Fig. 5. Here, contrary to the case of $\mathrm{MgB}_{2}$, both healing lengths decrease with respect to the standard GL $\xi$, and therefore the ratio $\xi_{1} / \xi_{2}$ is smaller than in $\mathrm{MgB}_{2}$. However, since in quantum confined systems, either nanofilms or pancake fermionic condensates, the exact position of the bands with respect to the Fermi level (and therefore the $v_{1} / v_{2}$ ratio) can be tuned the discrepancy between the corresponding healing lengths can be further enlarged.

\section{CONCLUSIONS}

To summarize, we demonstrated on examples of several relevant superconducting materials that characteristic length scales of coupled condensates in two- or multiband samples are in general different from each other in the domain of the extended Ginzburg-Landau theory. Although we do not show it explicitly, we mention here again that the extended model is not more complicated than its standard predecessor - it does contain more equations, but the coupling of those equations is realized in a computationally friendly manner. This makes our model an excellent tool for further studies of two-band systems, where one expects a plethora of unique physical effects emerging from the competition of different characteristic length scales.

\section{ACKNOWLEDGMENT}

This work was supported by the Flemish Science Foundation (FWO-Vl), the Belgian Science Policy (IAP), and the ESF-INSTANS network. 
${ }^{1}$ V. L. Ginzburg and L. D. Landau, Sov. Phys. JETP 20, 1064 (1950).

${ }^{2}$ V. G. Kogan and J. Schmalian, Phys. Rev. B 83, 054515 (2011).

${ }^{3}$ A. E. Koshelev and A. A. Golubov, Phys. Rev. Lett. 90, 177002 (2003).

${ }^{4}$ A. E. Koshelev and A. A. Golubov, Phys. Rev. Lett. 92, 107008 (2004).

${ }^{5}$ M. Silaev and E. Babaev, e-print arXiv:1102.5734v1.

${ }^{6}$ M. R. Eskildsen, M. Kugler, S. Tanaka, J. Jun, S. M. Kazakov, J. Karpinski, and Ø. Fischer, Phys. Rev. Lett. 89, 187003 (2002).

${ }^{7}$ S. Serventi, G. Allodi, R. De Renzi, G. Guidi, L. Romanò, P. Manfrinetti, A. Palenzona, Ch. Niedermayer, A. Amato, and Ch. Baines, Phys. Rev. Lett. 93, 217003 (2004).

${ }^{8}$ A. Shibata, M. Matsumoto, K. Izawa, Y. Matsuda, S. Lee, and S. Tajima, Phys. Rev. B 68, 060501(R) (2003).

${ }^{9}$ A. A. Shanenko, M. V. Milošević, F. M. Peeters, and A. V. Vagov, Phys. Rev. Lett. 106, 047005 (2011).

${ }^{10}$ E. Babaev and M. Silaev, e-print arXiv:1105.3756; V. G. Kogan and J. Schmalian, e-print arXiv:1105.5090.

${ }^{11}$ V. V. Moshchalkov, M. Menghini, T. Nishio, Q. H. Chen, A. V. Silhanek, V. H. Dao, L. F. Chibotaru, N. D. Zhigadlo, and J. Karpinski, Phys. Rev. Lett. 102, 117001 (2009).

${ }^{12}$ A. Chaves, L. Komendová, M. V. Milošević, J. S. Andrade Jr., G. A. Farias, and F. M. Peeters, Phys. Rev. B 83, 214523 (2011).

${ }^{13}$ M. E. Zhitomirsky and V.-H. Dao, Phys. Rev. B 69, 054508 (2004); R. Geurts, M. V. Milošević, and F. M. Peeters, ibid. 81, 214514 (2010).
${ }^{14}$ Y. Guo, Y.-F. Zhang, X.-Y. Bao, T. Z. Han, Z. Tang, L. X. Zhang, W. G. Zhu, E. G. Wang, Q. Niu, Z. Q. Qiu, J. F. Jia, Z. X. Zhao, and Q. K. Xue, Science 306, 1915 (2004).

${ }^{15}$ D. Eom, S. Qin, M. Y. Chou, and C. K. Shih, Phys. Rev. Lett. 96, 027005 (2006).

${ }^{16}$ S. Qin, J. Kim, Q. Niu, and C.-K. Shih, Science 324, 1314 (2009).

${ }^{17}$ T. Zhang, P. Cheng, W.-J. Li, Y. J. Sun, G. Wang, X. G. Zhu, K. He, L. L. Wang, X. C. Ma, X. Chen, Y. Y. Wang, Y. Liu, H. Q. Lin, J. F. Jia, and Q. K. Xue, Nat. Phys. 6, 104 (2010).

${ }^{18}$ P. Dyke, E. D. Kuhnle, S. Whitlock, H. Hu, M. Mark, S. Hoinka, M. Lingham, P. Hannaford, and C. J. Vale, Phys. Rev. Lett. 106, 105304 (2011)

${ }^{19}$ A. A. Golubov, J. Kortus, O. V. Dolgov, O. Jepsen, Y. Kong, O. K. Andersen, B. J. Gibson, K. Ahn, and R. K. Kremer, J. Phys. Condens. Matter 14, 1353 (2002).

${ }^{20}$ V. G. Kogan, C. Martin, and R. Prozorov, Phys. Rev. B 80, 014507 (2009).

${ }^{21}$ H. Kim, M. A. Tanatar, Y. J. Song, Y. S. Kwon, and R. Prozorov, Phys. Rev. B 83, 100502(R) (2011).

${ }^{22}$ K. Cho, H. Kim, M. A. Tanatar, Y. J. Song, Y. S. Kwon, W. A. Coniglio, C. C. Agosta, A. Gurevich, and R. Prozorov, Phys. Rev. B 83, 060502(R) (2011).

${ }^{23}$ A. Gurevich, Phys. Rev. B 82, 184504 (2010).

${ }^{24}$ A. A. Shanenko, M. D. Croitoru, A. Vagov, and F. M. Peeters, Phys. Rev. B 82, 104524 (2010).

${ }^{25}$ C. J. Thompson and J. M. Blatt, Phys. Lett. 5, 6 (1963).

${ }^{26}$ E. H. Hwang, S. Das Sarma, and M. A. Stroscio, Phys. Rev. B 61, 8659 (2000). 